\title{
Microscopy automation on a budget: capturing high-speed multi-labelled events with LED Illumination Systems
}

www.coolled.com

Capturing fast cellular processes with widefield fluorescence microscopy and multiple fluorophores has historically required the addition of motorised components. The light source has often been overlooked as an affordable means of achieving multi-wavelength imaging, but LEDs have changed the game.

Here we compare typical configurations for automated illumination systems in terms of speed, cost and signal-to-noise ratio (i.e. image contrast). We also explore how to transform a manual microscope into a powerful automated imaging system by capitalising on LED switching speeds, with lower cost filters and minimal requirement for expensive motorised components.

\section{TYPICAL APPROACH}

Many widefield fluorescence microscope systems include white light sources, such as mercury or metal halide lamps, or "white" LED illumination systems. Automated wavelength selection usually involves a motorised filter cube turret with single-band filters matching each individual fluorophore. Although this achieves high signal-to-noise ratio, relatively long switching times limit dynamic observations of multi-labelled samples to the order of seconds. This also relies on costly investment when upgrading a manual microscope.

\section{HIGH-SPEED IMAGING WITH A WHITE LIGHT SOURCE}

Faster observation can be achieved at a higher price point with high-speed external filter wheels and the following filter configurations:

- Pinkel filter set: single-band excitation filter; multi-band emission filter and dichroic mirror

- Sedat filter set: single-band excitation and emission filters; multi-band dichroic mirror

Whilst a Sedat filter set provides the highest signal-to-noise ratio, it requires two expensive filter wheels and extra filters. ${ }^{1}$ In terms of speed, filter wheels take around 55-100 ms to rotate to a new filter position.

\section{HIGH-SPEED IMAGING WITH A CONTROLLABLE LED ILLUMINATION SYSTEM}

Being solid-state, LEDs can be switched on and off electronically at exceptionally high speeds. More advanced LED illumination systems take advantage of this and can offer high speeds and a high signal-to-noise ratio at lower cost.

Using multi-band filters with LED illumination systems featuring individual channel selection overcomes latency associated with motorisation, for a fast and low-cost approach to multi-labelled imaging. However, bleedthrough and reduced signal-to-noise ratio can occur if multi-band filters are not chosen carefully. To overcome this, some LED illumination systems (such as the CoolLED $p E-300$ ultra, $p E-340^{\text {fura }}$ and $p E-800$ ) allow individual excitation filters (from a Pinkel filter set) to enhance the signal-to-noise ratio (Figure 1). Although the increased signal-tonoise ratio of a Sedat filter set is not possible, the slight compromise in image quality comes at the gain of significant speeds at a much lower price point.

\section{TAKING SPEED ONE STEP FURTHER} As with any peripheral device, USB serial communications and computer operating

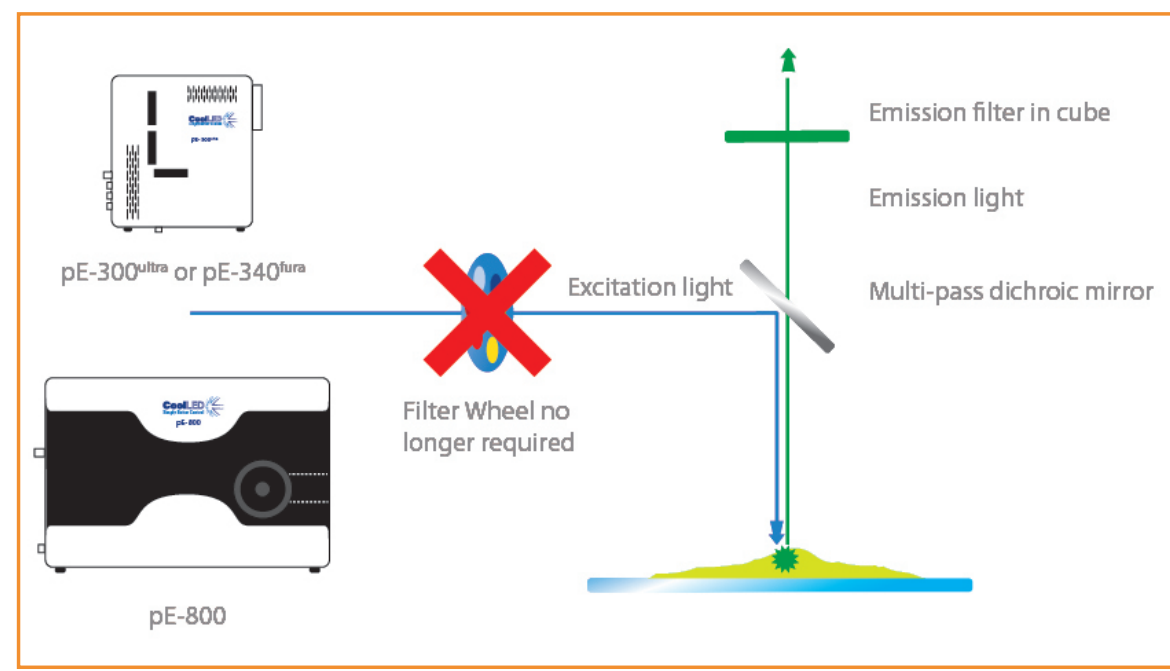

Figure 1. Capturing fast events with LED illumination systems. Individual LED channel switching and inline excitation filters with the CoolLED pE-300ultra, pE-340 fura or pE-800 allow a Pinkel filter configuration to replace the cost and latency of a filter wheel. This enables high-speed, high-contrast and cost-effective imaging of multi-labelled samples. 
systems can introduce latencies, potentially reducing switching speeds by as much as $100 \mathrm{~ms}$.

To capitalise on the maximum speed of LEDs, we recommend electronic control via TTL which offers switching speeds as fast as $<7 \mu$ s. While TTL often requires costly equipment, Sequence Runner is a feature which uses the TTL output signal available on most scientific cameras and cycles though LEDs in a user-selected sequence. This simple and cost-effective approach provides fast switching speeds precisely synchronised to the camera exposure. ${ }^{2}$

Limiting unnecessary sample illumination taking place outside of the camera exposure time (which is also known as "illumination overhead") minimises phototoxicity and photobleaching. With improvements in cell viability resulting from the former, this achieves not only insightful but accurate data. ${ }^{3}$

\section{QUICK COMPARISON}

The table below summarises the configurations discussed, using an example of an automated three-wavelength system.

\begin{tabular}{|c|c|c|}
\hline Configuration & Benefits & Drawbacks \\
\hline $\begin{array}{l}\text { White light source with external high-speed } \\
\text { filter wheels and Sedat filters }\end{array}$ & $\begin{array}{l}\text { Speed: improved temporal resolution } \\
(\sim 55 \text { ms switching time }) \\
\text { Signal-to-noise: high image contrast }\end{array}$ & $\begin{array}{l}\text { Cost: two expensive high-precision } \\
\text { filter wheels required for best temporal } \\
\text { resolution } \\
\text { Vibration artefacts possible }\end{array}$ \\
\hline $\begin{array}{l}\text { White light source with external high-speed } \\
\text { filter wheel and Pinkel filters }\end{array}$ & $\begin{array}{l}\text { Speed: improved temporal resolution } \\
\text { ( } 55 \text { ms switching time) } \\
\text { Signal-to-noise: high, although Sedat filter } \\
\text { set is higher }\end{array}$ & $\begin{array}{l}\text { Cost: Less than with a Sedat filter set, but } \\
\text { an expensive high-precision filter wheel is } \\
\text { required for best temporal resolution } \\
\text { Vibration artefacts possible }\end{array}$ \\
\hline $\begin{array}{l}\text { Controllable LED Illumination System } \\
\text { such as the CoolLED pE-300wite and full } \\
\text { multi-band filter set; with USB control }\end{array}$ & $\begin{array}{l}\text { Speed: no slow motorised parts } \\
\text { Cost: no expensive motorised filter wheel } \\
\text { Low risk/no vibration artefacts }\end{array}$ & $\begin{array}{l}\text { Signal-to-noise: increased potential for } \\
\text { bleed-through and reduced image contrast } \\
\text { with full multi-band filter configuration, } \\
\text { requiring image filtering }\end{array}$ \\
\hline $\begin{array}{l}\text { Controllable LED Illumination System such } \\
\text { as the CoolLED pE-300ultra, pE-340fura or } \\
\text { pE-800 and Sequence Runner, with Pinkel } \\
\text { filter set and TTL control }\end{array}$ & $\begin{array}{l}\text { Speed: super-fast }(<7 \mu \text { s). Minimal } \\
\text { phototoxicity and photobleaching } \\
\text { Cost: inexpensive automated imaging } \\
\text { system; no need for sophisticated } \\
\text { software } \\
\text { Signal-to-noise: high contrast }\end{array}$ & Requires camera with TTL-out \\
\hline
\end{tabular}

\section{CONCLUSION}

Many configurations are possible when seeking the optimal balance between speed, cost and contrast. However, reaching the highest temporal resolution requires a controllable LED illumination system which can achieve switching speeds of $<7 \mu$ s. These speeds may be achieved with a full multi-band filter set which also saves costs over traditional motorised filter wheels and turrets. Alternatively, individual LED channel control and inline single-band excitation filters overcome the drawbacks of bleed-through and achieve a high signal-to-noise ratio.

Sequence Runner further simplifies individual channel control and reduces cost, making it possible to set up multi-colour high-speed imaging experiments with high signal-to-noise via a single TTL. Not only does this increase temporal resolution, but the tight synchronisation has the added benefit of enhancing data accuracy through minimised phototoxicity and photobleaching.

The light source might seem like an unlikely enabler for high-speed imaging, but the level of control that comes with solid-state LEDs is a world away from traditional mercury and metal halide lamps.

\section{References}

1. Erdogan T. (2006). New optical filters improve high-speed multicolor fluorescence imaging. Biophotonics International 13, 34-39.

2. CoolLED (2018). Benefits of synchronising LED illumination systems to a camera. BioTechniques 65(6). https://doi.org/10.2144/btn-2018-2012

3. Kiepas A et al. (2020). Optimizing live-cell fluorescence imaging conditions to minimize phototoxicity. Journal of Cell Science 133(4), jcs242834. https://doi.org/10.1242/jcs.242834
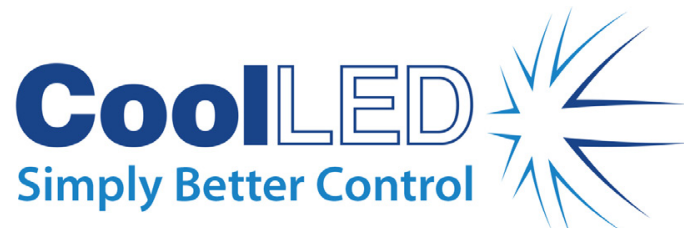